\title{
Safety of benzodiazepines and opioids in very severe respiratory disease: national prospective study
}

\author{
(c) (1) (8) OPEN ACCESS
}

\author{
Magnus P Ekström medical doctor, research fellow ${ }^{12}$, Anna Bornefalk-Hermansson biostatistician ${ }^{3}$, \\ Amy P Abernethy associate professor ${ }^{45}$, David C Currow professor ${ }^{5}$
}

${ }^{1}$ Department of Clinical Sciences, Division of Respiratory Medicine and Allergology, Lund University, SE-221 00 Lund, Sweden; ${ }^{2}$ Department of Medicine, Blekinge Hospital, SE-37185 Karlskrona, Sweden; ${ }^{3}$ Uppsala Clinical Research Center, Uppsala University Hospital, SE-752 37 Uppsala, Sweden; ${ }^{4}$ Division of Medical Oncology, Department of Medicine, Duke University Medical Center, Durham, USA; ${ }^{5}$ Discipline, Palliative and Supportive Services, Flinders University, SA-5041 Adelaide, Australia

\begin{abstract}
Objective To evaluate the safety of benzodiazepines and opioids in patients with very severe chronic obstructive pulmonary disease (COPD).

Design Population based longitudinal consecutive cohort study. Setting Centres prescribing long term oxygen therapy in Sweden.

Patients 2249 patients starting long term oxygen therapy for COPD in Sweden between 2005 and 2009 in the national Swedevox Register.

Main outcome measures Effects of benzodiazepines and opioids on rates of admission to hospital and mortality, adjusted for age, sex, arterial blood gases, body mass index (BMI), performance status, previous admissions, comorbidities, and concurrent drugs.

Results 1681 (76\%) patients were admitted to hospital, and 1129 (50\%) died under observation. No patient was lost to follow-up.

Benzodiazepines and opioids were not associated with increased admission: hazard ratio 0.98 ( $95 \%$ confidence interval, 0.87 to 1.10 ) and 0.98 ( 0.86 to 1.10$)$, respectively. Benzodiazepines were associated with increased mortality $(1.21,1.05$ to 1.39$)$ with a dose response trend. Opioids also had a dose response relation with mortality: lower dose opioids ( $\leq 30 \mathrm{mg}$ oral morphine equivalents a day) were not associated with increased mortality $(1.03,0.84$ to 1.26$)$ in contrast with higher dose opioids (1.21, 1.02 to 1.44). Concurrent benzodiazepines and opioids in lower doses were not associated with increased admissions $(0.86$, 0.53 to 1.42$)$ or mortality $(1.25,0.78$ to 1.99$)$. Associations were not modified by being naive to the drugs or by hypercapnia.
\end{abstract}

Conclusions Lower dose opioids are not associated with increased admissions or deaths in patients with COPD and might be safe for symptom reduction in severe respiratory disease.

\section{Introduction}

Breathlessness is a major cause of impaired activity and quality of life, affecting as many as a fifth of people aged over $65 .^{12}$ Chronic obstructive pulmonary disease (COPD) is a major cause of breathlessness, morbidity, and mortality. Worldwide, more than 300 million people have COPD, many of whom have breathlessness that affects their daily life for many years. ${ }^{23}$ Breathlessness predicts mortality in COPD, to a stronger degree than impairment of lung function. ${ }^{4}$ The burden of breathlessness increases with increasing age and severity of the respiratory disease; $98 \%$ of patients with end stage respiratory disease experience breathlessness, which persists at rest or on minimal exertion, despite optimal treatment of the underlying disease (chronic refractory breathlessness). ${ }^{5}$ Patients with severe COPD are more breathless than patients with advanced lung cancer and are breathless for longer periods of time. ${ }^{6}$

Randomised trials have shown that oral sustained release morphine can relieve chronic refractory breathlessness. ${ }^{78}$ Whether benzodiazepines reduce breathlessness is not clear, and their safety in this setting is unknown. ${ }^{9}$ Benzodiazepines are also used to treat anxiety and opioids to treat pain, conditions that are highly prevalent in patients with severe COPD. ${ }^{10}$

A concern among clinicians is that benzodiazepines and opioids alone or in combination could cause adverse events, including respiratory depression, confusion, falls, and even premature death in patients with respiratory compromise. Risks might be higher in frail patients, especially if they have not previously been treated with either or both drugs, and in people with severe COPD and hypercapnia. These concerns are cited by clinicians as an obstacle to prescribing these drugs and might contribute to less than optimal management of breathlessness. ${ }^{11}{ }^{12}$ 
Safety data for benzodiazepines and opioids in patients with severe COPD are limited. A pooled analysis of prospective trials including 178 patients with refractory breathlessness, $49 \%$ of whom had COPD, found no serious adverse events or admissions to hospital associated with treatment with low doses of opioid. ${ }^{13}$ Studies of lower dose benzodiazepines and lower dose opioids in patients with COPD found not a single case of respiratory depression. ${ }^{914} 15$ These studies, however, were mostly small short term trials that included a limited number of selected patients with close follow-up. ${ }^{9}{ }^{14}$ Safety data from clinical practice are lacking. ${ }^{13}{ }^{14}$ Estimates implying a minimal increase in risk with low dose treatments would be informative in a presumed high risk population.

We therefore estimated the effects of benzodiazepines and opioids on the risk of admission to hospital and death in patients with respiratory failure attributable to COPD.

\section{Methods}

This was a nationwide prospective consecutive cohort study with longitudinal follow-up of patients starting long term oxygen therapy for COPD diagnosed by a physician and registered between 1 October 2005 and 30 June 2009 in the national Swedevox Register. The Swedevox Register covers about $85 \%$ of patients starting long term oxygen therapy for COPD in Sweden. ${ }^{16}$ To increase the specificity of the diagnosis, we included only patients aged 45 or older. The exclusion criterion was a diagnosis of lung cancer at the start of oxygen therapy (baseline). A previous study that used this database and details of the study design have been published elsewhere. ${ }^{17}$

We obtained data on comorbidity and hospital admission for four years before baseline from the National Patient Register for inpatient and outpatient care, which covers more than $99 \%$ of all admissions in the study period and about $80 \%$ of all hospital based outpatient care since 2001 in Sweden. ${ }^{18}$ Definitions of the comorbid diagnoses have been published elsewhere. ${ }^{17}$

Data on all dispensed prescriptions during outpatient care after 1 July 2005 were obtained from the Swedish Prescribed Drug Register. ${ }^{19}$ Drugs were categorised according to the Anatomical Therapeutic Chemical Classification System (ATC codes) ${ }^{20}$ as antidepressants (N06A), benzodiazepines (N05BA), weak opioids (N02AA59, N02AX02), strong opioids (N02A except weak), and sleeping pills (N05C), and as previously described. ${ }^{17}$ Vital status was obtained from the Swedish Causes of Death Register.

\section{Statistical analysis}

Data were described with basic descriptive statistics. Drug exposure was defined as at least one dispensed prescription during the 91 days before baseline. Exposure during follow-up was tabulated for patients classified as unexposed and exposed at baseline. Exposure status at baseline was a good proxy for subsequent use during follow-up. Exposure to benzodiazepines and opioids was coded dichotomously (treated $v$ non-treated), continuously as the baseline dose, and categorised into lower and higher dose treatment. The baseline dose was calculated as the mean dispensed WHO defined daily doses per day during the 91 day period before baseline.$^{20}$ Lower dose treatment was defined as $\leq 0.3$ defined daily doses/day ( $\leq 30 \mathrm{mg}$ oral morphine equivalents/day), ${ }^{20}$ as this is the current evidence based dose range for opioid treatment for chronic refractory breathlessness in COPD ${ }^{1421}$ The same cut off was used for benzodiazepines, as it corresponded to the median dose among the exposed.
Benzodiazepines (1625 dispensed prescriptions) included oxazepam (74\%), diazepam (17\%), and alprazolam (8\%). Sleeping pills were mainly zopiclone (46\%), propiomazine (22\%), zolpidem (18\%), and flunitrazepam (7\%). Opioids (1417 prescriptions) included the weak opioids tramadol (31\% of all opioids), codeine (19\%), and dextropropoxyphene (15\%), and the strong opioids oxycodone (15\%), morphine (11\%), and fentanyl (5\%). There were no recorded prescriptions of nebulised opioids. At baseline, 275 (12\%) patients used only weak opioids, $189(8 \%)$ used only strong opioids, and $45(2 \%)$ used both. The analysis was predefined to include the total opioid dose in morphine equivalents, as both weak and strong opioids could be associated with dose dependent adverse events in severe illness. Weak and strong opioids were also merged into a single opioid category to improve precision as subgroup analyses yielded similar estimates.

Potential confounders evaluated in the analyses at baseline included age, sex, arterial blood gas tension of oxygen $\left(\mathrm{PaO}_{2}\right)$ and carbon dioxide $\left(\mathrm{PaCO}_{2}\right)$ in patients breathing air, forced expiratory volume in one second $\left(\mathrm{FEV}_{1}\right)$, smoking status, body mass index (BMI), WHO performance status, ${ }^{22}$ Charlson comorbidity index, anxiety/depression, diabetes mellitus, number of cardiovascular diagnoses (cerebrovascular disease, heart failure, hypertension, ischaemic heart disease, peripheral artery disease, pulmonary embolism, or other circulatory disease), osteoporosis, renal failure, number of previous admission to hospital, treatment with antidepressants, sleeping pills, whether long term oxygen therapy was started in hospital, and the number of admissions within four years before baseline. Missing elements were imputed for $\mathrm{PaO}_{2}$ air $(\mathrm{n}=289), \mathrm{PaCO}_{2}$ air $(\mathrm{n}=301)$, $\operatorname{BMI}(n=701)$, and WHO performance status $(n=199)$, as detailed elsewhere. ${ }^{17}$ We selected the covariates to be included in the final models using personal knowledge and a backward stepwise regression method, keeping interactions and predictors with $0.05 \leq \mathrm{P}$ value $<0.15$ if their removal distorted the robustness of the model.

Drug effects on the rates of admission to hospital were expressed as subdistribution hazard ratios and estimated by using Fine-Gray regression, which accounts for the competing risk of death. ${ }^{23}$ For the analysis of admissions, the time under observation was from the first day of long term oxygen therapy in the community until the date of first admission to hospital for all causes, with censoring at withdrawal of long term oxygen therapy, death, or study end (31 December 2009). The final admission model was adjusted for WHO performance status, number of previous admissions, number of cardiovascular diagnoses, diabetes mellitus, osteoporosis, and treatment with oral glucocorticoids.

Associations with mortality were expressed as hazard ratios and estimated with Cox regression. For mortality, the observation time was from the start date of long term oxygen therapy until the date of death from all causes, with censoring at withdrawal of long term oxygen therapy or 31 December 2009. The final mortality model was adjusted for age, sex, $\mathrm{PaO}_{2}$ air, $\mathrm{PaCO}_{2}$ air, WHO performance status, BMI, anaemia, number of cardiovascular diagnoses, renal failure, and oral glucocorticoids. Sleeping pills did not predict admission to hospital or mortality or affect the estimates for benzodiazepines and opioids. Findings were similar when we excluded patients taking sleeping pills.

We evaluated interactions, by including interaction terms in the fully adjusted models, between the effect of benzodiazepines and opioids and concurrent treatment with both drugs, the presence of hypercapnia $\left(\mathrm{PaCO}_{2}\right.$ air $\left.>6.5 \mathrm{kPa}\right)$, being naive to these drugs (no exposure during the year before baseline), having 
comorbid anxiety/depression (ICD-9 (international classifications of diseases, ninth revision) codes: 296, 298, 300, 311; ICD-10 (10th revision): F30, F48), and comorbid injury (mainly fractures) (ICD-9: 800,829,830, 999; ICD-10: M84, S02, S12, S22, S32, S42, S52, S62, S72, S82, S92, T02, T10, $\mathrm{T} 12)$. We analysed interactions between drugs and comorbid anxiety/depression and injury to explore possible confounding by the underlying indications for benzodiazepines and opioids. We also evaluated possible adverse opioid effects in the subset of opioid naive patients with injury, who were more likely to receive opioids because of a similar indication (pain) and to have increased risk of adverse events because of the underlying injury and being naive to opioids.

We calculated $95 \%$ confidence intervals for all estimates. The assumption of proportional hazards in the regression models was assured with $\log$-log plots and time varying covariates. Statistical analyses were performed with Stata version 12.1 (StataCorp LP; College Station, TX, USA).

\section{Results}

We included 2249 patients (1410, 59\% women). As shown in table $1 \Downarrow$, benzodiazepines were used by $535(24 \%)$ patients and opioids by $509(23 \%)$, and $200(9 \%)$ were taking both types of drug at baseline.

Compared with non-users, patients taking benzodiazepines or opioids were more likely to be women and had lower functional status, higher $\mathrm{PaCO}_{2}$ on room air, and more previous admissions to hospital, oral glucocorticoids, osteoporosis, injuries, and depression/anxiety.

Exposure to benzodiazepines and opioids throughout follow-up was higher among patients with baseline exposure (table $2 \Downarrow$ ); the median percentage of exposed time during follow-up was $100 \%$ (interquartile range 88-100) for benzodiazepines and $100 \%$ (60-100) for opioids. Patients unexposed at baseline had low rates of exposed time during subsequent follow-up (table 2). $\Downarrow$

\section{Admission to hospital}

Long term oxygen therapy was started during an admission in 641 patients (29\%), of whom 606 were discharged after a median 3 (interquartile range 1-7) days; 35 (2\%) died in hospital and were excluded from the analysis. Of the included 2214 patients, 1681 (76\%) were admitted after a median 76 (26-222) days of follow-up. Death without admission occurred in 182 patients $(8 \%)$.

Treatment with benzodiazepines or opioids was not associated with increased rates of admission; subdistribution hazard ratios were 0.98 (95\% confidence interval 0.87 to 1.10$)$ and $0.98(0.86$ to 1.10$)$, respectively. There were no dose-response relations (table $3 \Downarrow$ ). Higher dose treatment $(>0.3$ defined daily doses/day) did not increase the rate of admission for benzodiazepines (subdistribution hazard ratio $0.90,0.76$ to 1.06 ) or for opioids (1.01, 0.86 to 1.18$)$. The figure shows the adjusted risks of admission $\Downarrow$.

Concurrent treatment with benzodiazepines and opioids was associated with a lower rate of admission, interaction (subdistribution hazard ratio $0.67,95 \%$ confidence interval 0.51 to $0.87 ; \mathrm{P}=0.003$ ). This interaction, however, was influenced mainly by WHO performance status. The risk associated with concurrent treatment showed a non-significant upward trend for WHO statuses 1 and 2 but decreased risks for WHO statuses 3 and 4 , indicating that the rates of admission were reduced by the degree of care given to immobilised patients, who were more likely to use both benzodiazepines and opioids. This interaction, therefore, might not reflect a clinically significant synergy.

There were no interactions between drug effects and the presence of hypercapnia (benzodiazepines $\mathrm{P}=0.26$; opioids $\mathrm{P}=0.14$ ), being naive to these drugs (benzodiazepines $\mathrm{P}=0.15$; opioids $\mathrm{P}=0.19$ ), or having comorbid anxiety/depression (benzodiazepines $\mathrm{P}=0.52$ ) or injury (opioids $\mathrm{P}=0.58$ ).

\section{Mortality}

During a median follow-up of 1.1 years (interquartile range 0.6-2.0 years), 138 (6\%) patients withdrew from long term oxygen therapy mainly because of improved oxygenation, and $1129(50 \%)$ patients died.

The figure shows the adjusted mortality estimates. $\Downarrow$ Benzodiazepines were associated with increased adjusted mortality (hazard ratio $1.21,95 \%$ confidence interval 1.05 to 1.39). There was a trend of a dose relation between higher benzodiazepine doses and increased mortality $(1.01,1.00$ to $1.03 ; \mathrm{P}=0.082$ ) for each increment in dose of 0.1 defined daily dose/day.

Opioids showed a linear dose-response association with increased mortality (hazard ratio $1.01,95 \%$ confidence interval 1.00 to $1.03 ; \mathrm{P}=0.038$ ) for each dose increment of 0.1 defined daily dose/day. Lower dose opioids ( $\leq 30 \mathrm{mg}$ of oral morphine equivalents/day) were not associated with increased mortality (1.03, 0.84 to 1.26 ) (table $3 \Downarrow$ ). Among opioid naive patients with comorbid injury $(n=175)$, opioid treatment was not associated with increased mortality $(0.93,0.43$ to 2.02$)$.

In a detailed analysis of the adjusted risks for each dose level of concurrent benzodiazepine and opioid, high dose treatments were associated with increased mortality, while lower dose treatments were not associated with increased risk of death (table $4 \Downarrow)$.

There was no evidence that the effects of benzodiazepines and opioids were modified by concurrent use $(\mathrm{P}=0.400)$, the presence of hypercapnia (benzodiazepines $\mathrm{P}=0.26$; opioids $\mathrm{P}=0.15$ ), being naive to these drugs (benzodiazepines $\mathrm{P}=0.22$; opioids $\mathrm{P}=0.25$ ), having comorbid anxiety/depression (benzodiazepines $\mathrm{P}=0.34$ ) or previous injury (opioids $\mathrm{P}=0.53$ ).

\section{Sensitivity analysis}

To account for the severity of any underlying disease and possible misclassification of drug exposure because of time spent in hospital, ${ }^{24}$ we adjusted the models for the amount (quarters) of time in hospital during the 91 days before baseline. Thus, drug effects were estimated for patients with similar chances of becoming exposed. The estimates were consistent with the main analysis. Neither benzodiazepines nor opioids were associated with increased admission. Opioids in lower doses were not associated with increased risks of death (hazard ratio $1.00,95 \%$ confidence interval 0.81 to 1.22 ), as shown in the appendix.

\section{Discussion Principal findings}

In this national prospective study, treatment with lower dose opioids was not associated with an increased risk of admission to hospital or death in patients with COPD who were dependent on oxygen, regardless of whether the patient was naive to opioids, concurrently used benzodiazepines, or had hypercapnia. Treatment with benzodiazepines also did not affect the rate of admission but was associated with a modest increase in 
mortality. Though this association cannot be interpreted as cause and effect, the association remained after we adjusted for known or plausible confounders.

\section{Comparison with other studies}

Our results are important because evolving data support the role of lower dose opioids in treating breathlessness that is refractory to treatment focused on the cause. The present population based data suggest that opioids are safe when prescribed at lower doses. Clinical concern about the association between low dose opioids and respiratory depression was not supported, as we found no increases in admissions to hospital or mortality in this consecutive cohort. Benzodiazepines, which have an unclear role in the management of refractory breathlessness, have a safety profile that is less clear, as shown in this study.

The lack of associations between low dose opioids and increased risks of admissions and death is consistent with the limited previous data. ${ }^{722125}$ A pooled analysis of randomised controlled trials including 178 patients reported no serious adverse events and found no published case reports of respiratory depression, admission, or death attributable to regular lower dose opioids in patients with COPD. ${ }^{13}$ One of four studies that analysed blood gases found a clinically non-significant change in $\mathrm{PaO}_{2}$ and $\mathrm{PaCO}_{2}$ but no events of hypoventilation in relation to lower dose opioids. ${ }^{7}$ Studies in palliative care found no associations between opioids and increased rates of respiratory depression ${ }^{25}$ or death. ${ }^{26}$ In a large meta-analysis of parenteral opioids in postoperative patients, in whom the highest rates of toxicity are likely to be seen, the rate of hypoventilation after $10 \mathrm{mg}$ intramuscular morphine was $0.6 \%$ and similar to that of placebo. ${ }^{27}$

Similarly, lower dose benzodiazepines have not been associated with serious adverse events or impaired blood gases in randomised controlled trials. ${ }^{9}{ }^{28}$ In observational studies, benzodiazepines have been associated with increased risk of admission to hospital because of falls ${ }^{29}$ and of developing more frequent and severe pneumonia. ${ }^{30}$ In the present study, people taking both benzodiazepines and opioids were less likely to be admitted to hospital. This probably reflects that concurrent treatment was more common among severely ill patients, who might have received healthcare interventions such as advance care planning and admittance to nursing homes, which could decrease the need of subsequent admissions to hospital. Observational data on benzodiazepines and mortality in the general population are conflicting. ${ }^{31}$ Benzodiazepines have been associated with increased mortality in people admitted to nursing homes. ${ }^{32}$ Our finding that concurrent treatment with benzodiazepines and higher dose opioids was associated with increased mortality might be a true adverse effect or reflect more severe underlying disease that requires higher doses to relieve symptoms. The associations persisted in the multiple regression analyses and were not modified by diagnosed anxiety/depression or injury. The dose level analysis suggested that the increased mortality is related to concurrent treatment with higher dose benzodiazepine or opioid treatment and that concurrent lower dose treatment with opioids might be safe (table 4). $\Downarrow$

\section{What this study adds}

This study extends previous observations in several important ways. By estimating adverse drug effects in a relevant high risk population in clinical practice it supports, firstly, that lower dose opioids are not associated with increased risk of admission to hospital or death, and might be safe for symptomatic treatment even in patients with severe and end stage COPD. It also suggests that benzodiazepines and higher dose opioids are associated with modestly increased risks of mortality, especially when used concurrently. The possibility of true causal effects, however, needs to be validated by prospective observational and randomized trials.

\section{Strengths and limitations of the study}

A major strength of the present study is its inclusion of a large population based consecutive cohort of old severely ill patients with respiratory failure caused by COPD, many of whom had hypercapnia. Sweden has an integrated publicly financed health system that provides care independently of the patient's health insurance and financial status. No patient was lost to follow-up. Analyses were adjusted for major confounders, including comorbidity and concomitant drug treatment, and the findings probably have excellent applicability to current clinical practice in similar settings around the world.

One limitation is that we cannot rule out residual confounding from unmeasured covariates and from the indications for benzodiazepines and opioids because of the absence of randomisation. ${ }^{33}$ This bias, however, would tend to overestimate the risk of adverse events. We dealt with confounding by indication by restricting the analysis to patients with very severe oxygen dependent COPD and by adjusting for markers of the severity of underlying disease, comorbidity, and concomitant drug treatments. Another possible limitation is that dispensed prescriptions might not be equivalent to consumed drugs. Baseline exposure, however, was a good proxy of drug use during subsequent follow-up. Most exposed patients filled regular prescriptions, which supports the assumption that they actually took the drugs. A potential source of bias is that benzodiazepines and opioids might have been preferentially given to patients who had used and tolerated them before the start of the study, which could potentially underestimate harmful effects. The fact that drug effects were similar between patients who were naive to these drugs and patients with previous exposure, however, makes this less likely. The apparent hazards of higher dose benzodiazepines and opioids might be overestimated because of increased prescription and need for higher doses in patients in the terminal phase of their life limiting illness.

\section{Conclusions and clinical implications}

The evidence generated indicates that there is a cohort of patients with severe COPD who seem to tolerate regular lower dose opioids in a way that is likely to deliver a net symptomatic benefit. The level of follow-up and healthcare given to patients might influence the outcomes, and our findings should be interpreted in the context of current levels of clinical contact.

For clinicians, this study further supports the safety of regular low dose systemic opioids to reduce breathlessness in severely ill patients with respiratory compromise and

hypercapnia-patients who have an immense need for relief of symptoms. ${ }^{1510}$ The need for higher opioid doses for symptomatic control should be balanced against the possible increased risk of adverse events. Our study supports the suggestion that benzodiazepines should not be the first line treatment for breathlessness in those with respiratory failure, given the unclear evidence of net clinical benefit. ${ }^{9}$

Benzodiazepines and opioids can be safely combined in lower doses. All treatments assume adequate follow-up of the patient's clinical condition and symptoms, including proper prophylaxis and treatment for expected effects such as opioid related constipation. ${ }^{8}{ }^{14}$ The approach for chronic breathlessness is no 
different from that of opioid treatment for pain. ${ }^{14}$ Sustained release morphine should be considered as a first line treatment and should be initiated at a low dose regularly and titrated upward over days and weeks, balancing beneficial and adverse effects. ${ }^{14}{ }^{34}$ Titration up to $30 \mathrm{mg}$ morphine daily might safely improve breathlessness in over $60 \%$ of patients, with a mean decrease of $35 \%$ in the intensity of breathlessness from the person's own baseline. ${ }^{21}$

In conclusion, benzodiazepines and higher dose opioids were associated with increased adjusted mortality, whereas lower dose opioids were not associated with increased risk of hospital admission or death in patients with respiratory failure associated with COPD.

We thank Kerstin Ström, founder of the Swedevox register, and all the doctors and nurses who cared for the patients.

Contributors: MPE and AB-H acquired the data. MPE analysed and interpreted the data and is guarantor. All authors drafted the article, revised it for important intellectual content, and approved the final version.

Funding: This study was funded by the Research Council of Blekinge and the Swedish Heart-Lung Foundation.

Competing interests: All authors have completed the ICMJE uniform disclosure form at www.icmje.org/coi_disclosure.pdf and declare: no support from any organisation for the submitted work; no financial relationships with any organisations that might have an interest in the submitted work in the previous three years; no other relationships or activities that could appear to have influenced the submitted work.

Ethical approval: The study was approved by the Lund University research ethics committee (157/2007 and 350/2008), the Swedish National Board of Health and Welfare, and the Data Inspection Board. All patients gave their informed consent to participate.

Transparency declaration: MPE affirms that the manuscript is an honest, accurate, and transparent account of the study being reported; that no important aspects of the study have been omitted; and that any discrepancies from the study as planned have been explained.

Data sharing: Group level data, statistical code, and full details of the exploratory secondary analyses are available from the corresponding author. Additional data are not available as patient consent for data sharing was not obtained.

1 Currow DC, Plummer JL, Crockett A, Abernethy AP. A community population survey of Currow DC, Plummer JL, Crockett A, Abernethy AP. A community population survey of
prevalence and severity of dyspnea in adults. J Pain Symptom Manage 2009;38:533-45. prevalence and severity of dyspnea in adults. J Pain Symptom Manage 2009;38:533-45.
Parshall MB, Schwartzstein RM, Adams L, Banzett RB, Manning HL, Bourbeau J, et al. An official American Thoracic Society statement: update on the mechanisms, assessment, and management of dyspnea. Am J Respir Crit Care Med 2012;185:435-52.

3 Vos T, Flaxman AD, Naghavi M, Lozano R, Michaud C, Ezzati M, et al. Years lived with disability (YLDs) for 1160 sequelae of 289 diseases and injuries 1990-2010: a systematic analysis for the Global Burden of Disease Study 2010. Lancet 2012;380:2163-96.

4 Nishimura K, Izumi T, Tsukino M, Oga T. Dyspnea is a better predictor of 5 -year survival than airway obstruction in patients with COPD. Chest 2002;121:1434-40.

5 Elkington $\mathrm{H}$, White $\mathrm{P}$, Addington-Hall J, Higgs R, Edmonds $\mathrm{P}$. The healthcare needs of chronic obstructive pulmonary disease patients in the last year of life. Palliat Med 2005;19:485-91.

6 Edmonds $\mathrm{P}$, Karlsen S, Khan S, Addington-Hall J. A comparison of the palliative care needs of patients dying from chronic respiratory diseases and lung cancer. Palliat Med 2001;15:287-95.

7 Jennings AL, Davies AN, Higgins JP, Gibbs JS, Broadley KE. A systematic review of the use of opioids in the management of dyspnoea. Thorax 2002;57:939-44.

8 Abernethy AP, Currow DC, Frith P, Fazekas BS, McHugh A, Bui C. Randomised, double blind, placebo controlled crossover trial of sustained release morphine for the management of refractory dyspnoea. BMJ 2003;327:523-8.
9 Simon ST, Higginson IJ, Booth S, Harding R, Bausewein C. Benzodiazepines for the relief of breathlessness in advanced malignant and non-malignant diseases in adults. Cochrane Database Syst Rev 2010;1:CD007354.

10 Solano JP, Gomes B, Higginson IJ. A comparison of symptom prevalence in far advanced cancer, AIDS, heart disease, chronic obstructive pulmonary disease and renal disease. $J$ Pain Symptom Manage 2006;31:58-69.

11 Young J, Donahue M, Farquhar M, Simpson C, Rocker G. Using opioids to treat dyspnea in advanced COPD: attitudes and experiences of family physicians and respiratory therapists. Can Fam Physician 2012;58:e401-7.

12 Goodridge D, Lawson J, Rocker G, Marciniuk D, Rennie D. Factors associated with opioid dispensation for patients with COPD and lung cancer in the last year of life: A retrospective analysis. Int J Chron Obstruct Pulmon Dis 2010;5:99-105.

13 Johnson MJ, Bland JM, Oxberry SG, Abernethy AP, Currow DC. Opioids for chronic refractory breathlessness: patient predictors of beneficial response. Eur Respir J 2013;42:758-66

14 Rocker G, Horton R, Currow D, Goodridge D, Young J, Booth S. Palliation of dyspnoea in advanced COPD: revisiting a role for opioids. Thorax 2009;64:910-15.

15 Allcroft P, Margitanovic V, Greene A, Agar MR, Clark K, Abernethy AP, et al. The role of benzodiazepines in breathlessness: a single site, open label pilot of sustained release morphine together with clonazepam. J Palliat Med 2013;16:741-4.

16 Strom K, Boe J. A national register for long-term oxygen therapy in chronic hypoxia: preliminary results. Eur Respir $J$ 1988;1:952-8.

17 Ekstrom MP, Bornefalk-Hermansson A, Strom KE. Effects of cardiovascular drugs on mortality in severe chronic obstructive pulmonary disease. Am J Respir Crit Care Med 2013;187:715-20.

18 Swedish National Board of Health and Welfare. Quality and contents of the Swedish hospital discharge register. Center for Epidemiology, 2008.

19 Wettermark B, Hammar N, Fored CM, Leimanis A, Otterblad Olausson P, Bergman U, et al. The new Swedish prescribed drug register-opportunities for pharmacoepidemiological research and experience from the first six months. Pharmacoepidemiol Drug Saf 2007;16:726-35.

20 WHO Collaborating Centre for Drug Statistics Methodology. Guidelines for ATC classification and DDD assignment 2011. WHO, 2010.

21 Currow DC, McDonald C, Oaten S, Kenny B, Allcroft P, Frith P, et al. Once-daily opioids for chronic dyspnea: a dose increment and pharmacovigilance study. J Pain Symptom Manage 2011;42:388-99

22 Oken MM, Creech RH, Tormey DC, Horton J, Davis TE, McFadden ET, et al. Toxicity and response criteria of the Eastern Cooperative Oncology Group. Am J Clin Oncol 1982;5:649-55.

23 Fine JP, Gray RJ. A proportional hazards model for the subdistribution of a competing risk. J Am Stat Assoc 1999;94:496-509.

24 Suissa S. Immeasurable time bias in observational studies of drug effects on mortality Am J Epidemiol 2008;168:329-35.

25 Clemens KE, Quednau I, Klaschik E. Is there a higher risk of respiratory depression in opioid-naive palliative care patients during symptomatic therapy of dyspnea with strong opioids? J Palliat Med 2008;11:204-16.

26 Lanken PN, Terry PB, Delisser HM, Fahy BF, Hansen-Flaschen J, Heffner JE, et al. An official American Thoracic Society clinical policy statement: palliative care for patients with respiratory diseases and critical illnesses. Am J Respir Crit Care Med 2008;177:912-27.

27 McQuay HJ, Moore RA. Postoperative analgesia and vomiting, with special reference to day-case surgery: a systematic review. Health Technol Assess 1998;2:1-236.

28 Stege G, Heijdra YF, van den Elshout FJ, van de Ven MJ, de Bruijn PJ, van Sorge AA, et al. Temazepam $10 \mathrm{mg}$ does not affect breathing and gas exchange in patients with severe normocapnic COPD. Respir Med 2010;104:518-24.

29 Huang AR, Mallet L, Rochefort CM, Eguale T, Buckeridge DL, Tamblyn R. Medication-related falls in the elderly: causative factors and preventive strategies. Drugs Aging 2012;29:359-76.

30 Obiora E, Hubbard R, Sanders RD, Myles PR. The impact of benzodiazepines on occurrence of pneumonia and mortality from pneumonia: a nested case-control and survival analysis in a population-based cohort. Thorax 2013;68:163-70.

31 Charlson F, Degenhardt L, McLaren J, Hall W, Lynskey M. A systematic review of research examining benzodiazepine-related mortality. Pharmacoepidemiol Drug Saf2009:18:93-103

32 Huybrechts KF, Rothman KJ, Silliman RA, Brookhart MA, Schneeweiss S. Risk of death and hospital admission for major medical events after initiation of psychotropic medications in older adults admitted to nursing homes. CMAJ 2011;183:E411-9.

33 Bosco JLF, Silliman RA, Thwin SS, Geiger AM, Buist DSM, Prout MN, et al. A most stubborn bias: no adjustment method fully resolves confounding by indication in observational studies. J Clin Epidemiol 2010;63:64-74

34 Currow DC, Quinn S, Greene A, Bull J, Johnson MJ, Abernethy AP. The longitudinal pattern of response when morphine is used to treat chronic refractory dyspnea. $J$ Palliat Med 2013;16:881-6.

Accepted: 17 January 2014

\section{Cite this as: BMJ 2014;348:9445}

This is an Open Access article distributed in accordance with the Creative Commons Attribution Non Commercial (CC BY-NC 3.0) license, which permits others to distribute, remix, adapt, build upon this work non-commercially, and license their derivative works on different terms, provided the original work is properly cited and the use is non-commercial. See: http://creativecommons.org/licenses/by-nc/3.0/. 


\section{What is already known on this topic}

Chronic breathlessness is common in patients with severe respiratory disease and can be relieved by opioids; benzodiazepines are commonly used to treat anxiety, which is common in patients with COPD

There are concerns that these drugs, alone or in combination, can cause respiratory depression, admission to hospital, and earlier death Safety data from clinical practice for benzodiazepines and opioids in people with severe COPD are lacking

\section{What this study adds}

Opioids in lower doses ( $\leq 30 \mathrm{mg}$ oral morphine equivalents/day) are not associated with increased hospital admissions or deaths in patients receiving long term oxygen therapy for COPD, whereas benzodiazepines and opioids in higher doses might increase mortality Lower dose opioids might be safe for reduction of symptoms in those with severe respiratory disease

\section{Tables}

Table 1/ Baseline characteristics according to treatment status of 2249 patients with oxygen dependent chronic obstructive pulmonary disease. Figures are means (SD) unless stated otherwise*

\begin{tabular}{lccc} 
Characteristic & Benzodiazepine (n=535) & Opioid (n=509) & Unexposed (n=1405) \\
Age (years) & $74.2(8.7)$ & $74.3(8.5)$ & $74.7(8.1)$ \\
\hline $\mathrm{No}(\%)$ of women & $383(72)$ & $360(71)$ & $667(47)$ \\
\hline $\mathrm{PaO}_{2}$ air (kPa) & $6.6(0.8)$ & $6.5(0.9)$ & $6.5(0.9)$ \\
\hline $\mathrm{PaCO}_{2}$ air (kPa) & $6.4(1.1)$ & $6.4(1.2)$ & $6.2(1.3)$ \\
\hline $\mathrm{FEV}_{1}(\%$ of predicted) & $30.0(14.7)$ & $33.2(16.1)$ & $34.7(18.2)$ \\
\hline No (\%) who had ever smoked & $492(92)$ & $472(93)$ & $1,323(94)$ \\
\hline Body mass index (BMI) & $23.6(6.5)$ & $24.8(6.6)$ & $23.9(6.1)$ \\
\hline No (\%) by WHO performance status: & & & \\
\hline $0-1$ & $192(36)$ & $180(35)$ & $704(50)$ \\
\hline$\geq 2$ & $288(54)$ & $275(54)$ & $582(41)$ \\
\hline Missing & $55(10)$ & $54(11)$ & $119(8)$ \\
\hline Median (IQR) No of previous admissions to hospital $\dagger$ & $6(3-11)$ & $7(4-11)$ & $4(2-7)$ \\
\hline No (\%) by other conditions/treatment: & & & \\
\hline Anaemia & $58(11)$ & $55(11)$ & $101(7)$ \\
\hline Cardiovascular diseases: & & & \\
\hline 0 & $151(28)$ & $130(26)$ & $525(37)$ \\
\hline 1 & $207(39)$ & $194(38)$ & $496(73)$ \\
\hline 2 & $120(22)$ & $117(23)$ & $262(19)$ \\
\hline$\geq 3$ & $57(11)$ & $68(13)$ & $122(9)$ \\
\hline Depression/anxiety & $108(20)$ & $65(13)$ & $75(5)$ \\
\hline Diabetes mellitus & $62(12)$ & $88(17)$ & $172(12)$ \\
\hline Injury & $156(29)$ & $187(37)$ & $217(15)$ \\
\hline Osteoporosis & $86(16)$ & $109(21)$ & $99(7)$ \\
\hline Renal failure & $23(4)$ & $27(5)$ & $59(4)$ \\
\hline Benzodiazepines & $535(100)$ & $200(39)$ & 0 \\
\hline Opioids & $200(37)$ & $509(100)$ & 0 \\
\hline Oral glucocorticoids & $371(69)$ & $325(64)$ & $810(58)$ \\
\hline & & & \\
\hline
\end{tabular}

IQR=interquartile range.

*Percentages might not sum to 100 because of rounding.

†ln four years before baseline. 
Table 2| Exposure to benzodiazepines and opioids in 2249 patients with oxygen dependent chronic obstructive pulmonary disease. Figures are numbers (percentage) unless stated otherwise

\begin{tabular}{|c|c|c|}
\hline & Benzodiazepines & Opioids \\
\hline \multicolumn{3}{|l|}{ Exposed at baseline } \\
\hline Total & $535(24)$ & $509(23)$ \\
\hline Low dose & $275(12)$ & $211(9)$ \\
\hline High dose & $260(12)$ & $298(13)$ \\
\hline Median (IQR) dose at baseline (DDD/day) & $0.27(0.11-0.66)$ & $0.37(0.18-0.73)$ \\
\hline Median (IQR) exposure during follow-up (\% of time) ${ }^{*}$ & $100(88-100)$ & $100(60-100)$ \\
\hline Drug naive (unexposed) in preceding 12 months & $68(4) \dagger$ & $75(4) \dagger$ \\
\hline \multicolumn{3}{|l|}{ Not exposed at baseline } \\
\hline Total & $1714(76)$ & $1740(77)$ \\
\hline Unexposed at baseline but exposed during follow-up & $474(21)$ & $509(23)$ \\
\hline Median (IQR) exposure during follow-up (\% of time) $)^{*}$ & $0(0-9)$ & $0(0-12)$ \\
\hline Drug naive (unexposed) in preceding 12 months & $1148(67) \dagger$ & $1082(63) \dagger$ \\
\hline
\end{tabular}

$\mathrm{DDD}=$ defined daily dose; $I Q R=$ interquartile range.

*Median percentage of exposed time during follow-up, calculated as sum of exposed 91 day periods divided by number of full 91 day periods during follow-up (from quarter before baseline until first event of withdrawal of long term oxygen therapy, death, or study end).

†Among patients with at least 12 months drug exposure data $(n=1704)$. 
Table 3| Benzodiazepines and opioids and adjusted risk of admission to hospital (subdistribution hazard ratio) and mortality (hazard ratio) in 2249 patients with oxygen dependent COPD*

\begin{tabular}{|c|c|c|}
\hline & \multicolumn{2}{|c|}{ Adjusted hazard ratio $(95 \% \mathrm{Cl})$} \\
\hline & Admission & Mortality \\
\hline \multicolumn{3}{|l|}{ Benzodiazepines: } \\
\hline Low dose & $1.05(0.90$ to 1.22$)$ & $1.18(0.99$ to 1.40$)$ \\
\hline High dose & 0.90 (0.76 to 1.06$)$ & $1.23(1.02$ to 1.48$)$ \\
\hline \multicolumn{3}{|l|}{ Opioids: } \\
\hline Low dose & $0.95(0.80$ to 1.14$)$ & $1.03(0.84$ to 1.26$)$ \\
\hline High dose & 1.01 (0.86 to 1.18$)$ & $1.21(1.02$ to 1.44$)$ \\
\hline \multicolumn{3}{|l|}{ Covariates } \\
\hline Age (/year) & - & $1.04(1.03$ to 1.05$)$ \\
\hline Male & - & $1.33(1.17$ to 1.51$)$ \\
\hline $\mathrm{PaO}_{2}$ air $(/ 1 \mathrm{kPa})$ & - & 0.92 (0.85 to 0.98$)$ \\
\hline $\mathrm{PaCO}_{2}$ air & - & $-\dagger$ \\
\hline \multicolumn{3}{|l|}{ Body mass index (BMI): } \\
\hline$<18.5$ & - & $1.36(1.15$ to 1.61$)$ \\
\hline $18.5-24.9$ & - & Ref \\
\hline $25-29.9$ & - & $0.71(0.59$ to 0.86$)$ \\
\hline$\geq 30$ & - & $0.80(0.64$ to 1.00$)$ \\
\hline \multicolumn{3}{|l|}{ WHO performance status: } \\
\hline 0 & Ref & Ref \\
\hline 1 & $1.02(0.83$ to 1.25$)$ & $1.03(0.75$ to 1.41$)$ \\
\hline 2 & 1.09 (0.89 to 1.34$)$ & 1.50 (1.10 to 2.06$)$ \\
\hline 3 & $0.90(0.70$ to 1.16$)$ & 2.34 (1.68 to 3.26$)$ \\
\hline 4 & $0.43(0.23$ to 0.84$)$ & 3.25 (1.97 to 5.38$)$ \\
\hline Missing & $1.10(0.85$ to 1.42$)$ & 1.35 (0.95 to 1.93$)$ \\
\hline $\begin{array}{l}\text { Previous admission in previous four years } \\
\text { (/admission) }\end{array}$ & $1.04(1.03$ to 1.05$)$ & - \\
\hline Anemia & - & 1.26 (1.03 to 1.52$)$ \\
\hline \multicolumn{3}{|l|}{ No of cardiovascular diseases: } \\
\hline 0 & Ref & Ref \\
\hline 1 & 1.03 (0.92 to 1.16$)$ & 1.24 (1.07 to 1.42$)$ \\
\hline 2 & 1.26 (1.09 to 1.45$)$ & $1.36(1.14$ to 1.61$)$ \\
\hline$\geq 3$ & 1.08 (0.89 to 1.32$)$ & $1.29(1.04$ to 1.61$)$ \\
\hline Depression/anxiety & - & - \\
\hline Diabetes mellitus & 1.26 (1.09 to 1.46$)$ & - \\
\hline Osteoporosis & 0.85 (0.72 to 1.01$)$ & - \\
\hline Renal failure & - & 1.34 (1.03 to 1.74$)$ \\
\hline Oral glucocorticoids & 1.35 (1.22 to 1.49$)$ & 1.18 (1.04 to 1.33$)$ \\
\hline
\end{tabular}

*Adjusted rates of admission and mortality were estimated with Fine-Gray regression and Cox regression, respectively. Low dose treatment was defined as $\leq 0.3$ defined daily dose/day and high dose as $>0.3$ defined daily dose/day. Estimates reported for all independent predictors included in final models. $+\mathrm{PaCO}_{2}$ air included as second degree polynomial (Wald $\mathrm{P}<0.001$ ); so linear hazard ratio is not reported. 
Table 4| Dose levels ${ }^{\star}$ of concurrent benzodiazepines and opioids and adjusted risk of admission to hospital (subdistribution hazard ratio) and mortality (hazard ratio)†

\begin{tabular}{lccc} 
& & \multicolumn{2}{c}{ Adjusted hazard ratio (95\% Cl) } \\
\cline { 2 - 4 } Benzodiazepine dose & Opioid dose & Admission & Mortality \\
\hline None & None & Ref & Ref \\
\hline None & Low & $1.08(0.89$ to 1.30$)$ & $1.03(0.81$ to 1.31$)$ \\
\hline Low & High & $1.15(0.94$ to 1.41$)$ & $1.13(0.90$ to 1.43$)$ \\
\hline Low & None & $1.16(1.00$ to 1.37$)$ & $1.11(0.90$ to 1.37$)$ \\
\hline Low & Low & $0.86(0.53$ to 1.42$)$ & $1.25(0.78$ to 1.99$)$ \\
\hline High & High & $0.92(0.65$ to 1.30$)$ & $1.68(1.17$ to 2.42$)$ \\
\hline High & None & $1.05(0.87$ to 1.28$)$ & $1.25(0.98$ to 1.57$)$ \\
\hline High & Low & $0.64(0.35$ to 1.18$)$ & $1.13(0.66$ to 1.92$)$ \\
\hline
\end{tabular}

${ }^{*}$ Categorised as none (untreated), low dose ( $\leq 0.3$ defined daily dose/day), and high dose ( $>0.3$ defined daily dose/day). †Rates of admission and mortality adjusted for covariates listed in table 3 .

$\ddagger$ Admission analysis included fewer patients, as patients who were in hospital during entire follow-up were excluded ( $n=35$ ). 


\section{Figure}

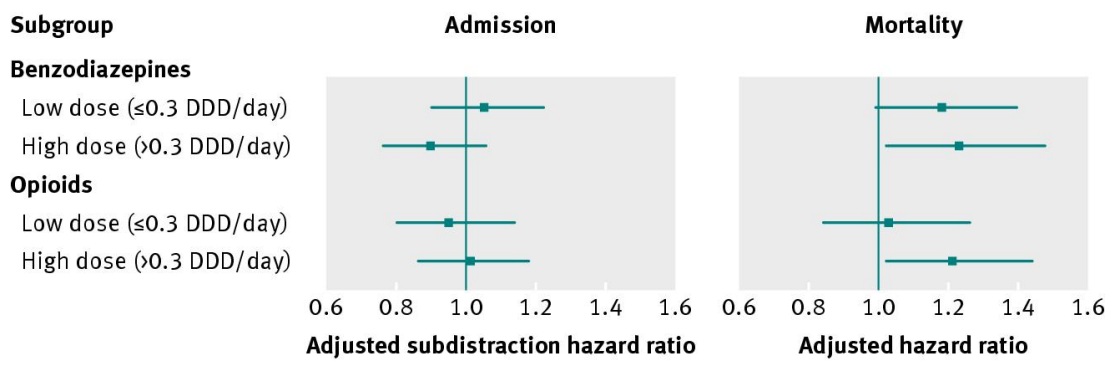

Adjusted hazard ratios with $95 \%$ confidence intervals. Rates of hospital admission rates adjusted for WHO performance status, number of previous admissions, number of cardiovascular diagnoses, diabetes mellitus, osteoporosis, and treatment with oral glucocorticoids. Mortality adjusted for age, sex, $\mathrm{PaO}_{2}$ air, $\mathrm{PaCO}_{2}$ air, $\mathrm{WHO}$ performance status, BMI, anaemia, number of cardiovascular diagnoses, renal failure, and oral glucocorticoids. DDD=defined daily dose 